PROCEEDINGS OF THE

AMERICAN MATHEMATICAL SOCIETY

Volume 133, Number 12, Pages 3687-3690

S 0002-9939(05)07933-5

Article electronically published on June 3, 2005

\title{
NONEXISTENCE OF SKEW LOOPS ON ELLIPSOIDS
}

\author{
MOHAMMAD GHOMI
}

(Communicated by Jon G. Wolfson)

\begin{abstract}
We prove that every $C^{1}$ closed curve immersed on an ellipsoid has a pair of parallel tangent lines. This establishes the optimal regularity for a phenomenon first observed by B. Segre. Our proof uses an approximation argument with the aid of an estimate for the size of loops in the tangential spherical image of a spherical curve.
\end{abstract}

A skew loop is a $C^{1}$ closed curve without parallel tangent lines immersed in Euclidean space. B. Segre [5] was the first to prove the existence of such curves, the first explicit construction appeared in [2, and Y.-Q. Wu 9] showed examples exist in each knot class. Segre also observed that sufficiently smooth skew loops do not exist on ellipsoids [3, Appdx. C]. This was an immediate consequence of a theorem of W. Fenchel [1], which states that the tangential spherical image, a.k.a. tantrix, of a (sufficiently smooth) closed spherical curve bisects the area of the sphere, when embedded. Fenchel's theorem in turn follows quickly from the GaussBonnet theorem, together with the fact that the total geodesic curvature of the tantrix of a spherical curve, when embedded, is zero [6]. But applying the GaussBonnet theorem requires that the tantrix be (at least) $C^{1}$. Hence the original curve should be $C^{2}$. Other proofs of Segre's observation [7, 8, which use Morse theory, also require $C^{2}$ regularity.

In this note we use an approximation argument to rule out the existence of skew loops on ellipsoids, without assuming extra regularity. This settles a question which had been raised in [3, Note 3.1]. The main obstacle here is that skew loops do not form an open subset in the space of loops. Indeed, small perturbations may create nearby parallel tangents. We overcome this problem by using the estimate provided in the following lemma. A mapping $T: \mathbf{S}^{1} \rightarrow \mathbf{S}^{2}$ is the tantrix of a $C^{1}$ immersion $\gamma: \mathbf{S}^{1} \simeq \mathbf{R} / 2 \pi \rightarrow \mathbf{R}^{3}$ provided $T(t)=\gamma^{\prime}(t) /\left\|\gamma^{\prime}(t)\right\|$. The following observation shows that the tantrix of a spherical loop may not contain small subloops:

Lemma. Let $T: \mathbf{S}^{1} \rightarrow \mathbf{S}^{2}$ be the tantrix of a spherical curve. Suppose that there are $t, s \in \mathbf{S}^{1}, t \neq s$, such that $T(t)=T(s)$. Then in each of the segments of $\mathbf{S}^{1}$ determined by $t$ and $s$ there exists a point, say $p$ and $q$ respectively, such that

$$
\operatorname{dist}_{\mathbf{S}^{2}}(T(t), T(p))=\frac{\pi}{2}=\operatorname{dist}_{\mathbf{S}^{2}}(T(t), T(q)) .
$$

Received by the editors April 21, 2004 and, in revised form, August 17, 2004.

2000 Mathematics Subject Classification. Primary 53A04, 53A05; Secondary 53C45, 52A15.

Key words and phrases. Tantrix, skew loop, ellipsoid, quadric surface.

The author's research was partially supported by NSF Grant DMS-0336455, and CAREER award DMS-0332333. 
Proof. Let $I$ and $J$ be the segments in $\mathbf{S}^{1}$ determined by $t$ and $s$. After a rotation, we may assume that $T(t)=\mathbf{k}=T(s)$, where $\mathbf{k}:=(0,0,1)$, the north pole of $\mathbf{S}^{2}$. Let $f: I \rightarrow \mathbf{R}$ be given by

$$
f(t):=\langle\gamma(t), \mathbf{k}\rangle
$$

where $\gamma$ is the parent curve of $T$, that is, $T(t)=\gamma^{\prime}(t) /\left\|\gamma^{\prime}(t)\right\|$, and define the equator of $\mathbf{S}^{2}$ as

$$
E:=\left\{x \in \mathbf{S}^{2} \mid\langle x, \mathbf{k}\rangle=0\right\} .
$$

Since $T(t)=\mathbf{k}=T(s)$, it follows that $\gamma(t), \gamma(s) \in E$ (a tangent vector of a sphere can be vertical only at the equator). Consequently, $f(t)=0=f(s)$. But, since $\gamma$ is orthogonal to $E$ at its end points, $f$ is not identically zero. So $f$ has a critical point $p$ in the interior of $I$; therefore,

$$
0=f^{\prime}(p)=\left\langle\gamma^{\prime}(p), \mathbf{k}\right\rangle=\left\|\gamma^{\prime}(p)\right\|\langle T(p), \mathbf{k}\rangle
$$

which yields $\langle T(p), \mathbf{k}\rangle=0$, or $T(p) \in E$. Thus,

$$
\operatorname{dist}_{\mathbf{S}^{2}}(T(t), T(p))=\frac{\pi}{2}
$$

Similarly there exists a point $q$ in $J$ whose spherical distance from $T(t)$ is $\pi / 2$.

Note 1. The above lemma implies that the total curvature of a spherical curve segment which has parallel tangents at its end points is at least $\pi$. Can one obtain lower bounds for total curvature of curves with parallel ends on other quadric surfaces? If so, then one could generalize the following theorem accordingly.

Now we are ready to prove the main result of this note:

Theorem. There are no skew loops on ellipsoids.

Proof. Since skew loops are affinely invariant, it is enough to rule out their existence on spheres. Let $\gamma: \mathbf{S}^{1} \rightarrow \mathbf{S}^{2}$ be a $C^{1}$ immersed loop, and let $\gamma_{i}$ be a family of $C^{2}$ immersed loops on $\mathbf{S}^{2}$ which converge to $\gamma$ with respect to the $C^{1}$ norm. Let $T$ be the tantrix of $\gamma$, and let $T_{i}$ be the tantrix of $\gamma_{i}$. Since there are no $C^{2}$ skew loops on $\mathbf{S}^{2}$, at least one of the following conditions holds for each $i$ :

1. $T_{i}\left(\mathbf{S}^{1}\right) \cap\left(-T_{i}\left(\mathbf{S}^{1}\right)\right) \neq \emptyset$.

2. There exist $t_{i}, s_{i} \in \mathbf{S}^{1}, t_{i} \neq s_{i}$, such that $T_{i}\left(t_{i}\right)=T_{i}\left(s_{i}\right)$.

Suppose that there exists a subsequence $\gamma_{i_{j}}$ of $\gamma_{i}$ all of whose elements satisfy condition 1 above. Then, since $T_{i}$ converges to $T$ (with respect to the $C^{0}$ norm), $T$ satisfies the same condition, which in turn yields that $\gamma$ is not skew, and we are done. So we may assume that there exists an integer $N$, such that condition 2 above is satisfied for all $i \geq N$. Then

$$
T(s)=T(t)
$$

where $t$ and $s$ are limit points of $t_{i}$ and $s_{i}$, respectively. Next recall that, by the above Lemma, there are points $p_{i}$ and $q_{i}$, in the interior of each of the segments of $\mathbf{S}^{1}$ determined by $t_{i}$ and $s_{i}$, such that

$$
\operatorname{dist}_{\mathbf{S}^{2}}\left(T_{i}\left(t_{i}\right), T_{i}\left(p_{i}\right)\right)=\frac{\pi}{2}=\operatorname{dist}_{\mathbf{S}^{2}}\left(T_{i}\left(t_{i}\right), T_{i}\left(q_{i}\right)\right) .
$$

Thus, if $p$ and $q$ are limits of $p_{i}$ and $q_{i}$, continuity of dist $\mathbf{S}^{2}$ yields that

$$
\operatorname{dist}_{\mathbf{S}^{2}}(T(t), T(p))=\frac{\pi}{2}=\operatorname{dist}_{\mathbf{S}^{2}}(T(t), T(q)) .
$$


In particular, $p \neq t$ and $q \neq t$. Now let $I_{i}$ and $J_{i}$ be segments of $\mathbf{S}^{1}$ determined by $t_{i}$ and $s_{i}$ such that $p_{i} \in I_{i}$ and $q_{i} \in J_{i}$, and let $I$ and $J$ be the limits of these segments, respectively. Then $p \in I$ and $q \in J$. Thus $p \neq t$ and $q \neq t$ imply that $I \neq t$ and $J \neq t$, respectively. Since $I$ and $J$ are each bounded by $t$ and $s$, this yields

$$
t \neq s
$$

Hence $\gamma$ is not skew.

Note 2. There is a simple argument for proving the nonexistence of $C^{1}$ skew loops $\gamma: \mathbf{S}^{1} \rightarrow \mathbf{S}^{2}$ provided we assume that $\gamma\left(\mathbf{S}^{1}\right)$ does not pass through any pairs of antipodal points of $\mathbf{S}^{2}$. To see this let $t, s \in \mathbf{S}^{1}$ be a pair of points which maximize $\operatorname{dist}_{\mathbf{S}^{2}}(\gamma(t), \gamma(s))$. Then the geodesic segment in $\mathbf{S}^{2}$ connecting $\gamma(s)$ and $\gamma(t)$ is orthogonal to $\gamma\left(\mathbf{S}^{1}\right)$ at these two points (here we use the assumption that $\gamma(t) \neq-\gamma(s))$. But every geodesic of $\mathbf{S}^{2}$ is a great circle, given by the intersection of $\mathbf{S}^{2}$ with a plane passing through the origin. Thus $\gamma\left(\mathbf{S}^{1}\right)$ is perpendicular, at $\gamma(t)$ and $\gamma(s)$, to this plane, which yields that $T(t)= \pm T(s)$.

The author and B. Solomon [3] have shown that, in addition to the ellipsoids, there are no $C^{2}$ skew loops on any quadric surfaces of positive curvature, and also have proved the converse. Further, S. Tabachnikov [7] has ruled out the existence of generic skew loops on any quadric surface - a result which generalizes to all dimensions (there are no generic skew branes on quadric hypersurfaces). Earlier J. H. White [8] had obtained the same result for spheres by studying the critical points of the intrinsic distances on the sphere. All these results assume $C^{2}$ regularity. But, as for the case of two-dimensional ellipsoids studied in this paper, we believe that $C^{1}$ regularity should be enough:

Conjecture. Any closed $C^{1}$-immersed submanifold $M^{n}$ of $\mathbf{R}^{n+2}$ has a pair of parallel tangent spaces if it lies on a quadric hypersurface.

In other words, there exists no skew branes on a quadric hypersurface. For results on the existence of skew submanifolds in arbitrary dimensions see [4].

\section{ACKNOWLEDGMENT}

The author thanks David Jerison for a stimulating conversation during a visit to MIT, where this problem was solved. Further, he is indebted to Bruce Solomon and Serge Tabachnikov for their interest in skew submanifolds and for pointing out errors in earlier drafts of this work.

\section{REFERENCES}

1. W. Fenchel, Über einen Jacobischen Satz der Kurventheorie. Tôhoku Math J., 39:95-97, 1934.

2. M. Ghomi, Shadows and convexity of surfaces. Ann. of Math. (2), 155(1):281-293, 2002. MR.1888801 (2003d:53006)

3. M. Ghomi and B. Solomon, Skew loops and quadric surfaces. Comment. Math. Helv. 77:767782, 2002. MR.1949113(2003m:53003)

4. M. Ghomi and S. Tabachnikov, Totally skew embeddings of manifolds. Preprint.

5. B. Segre, Global differential properties of closed twisted curves. Rend. Sem. Mat. Fis. Milano, 38:256-263, 1968. MR0240754 (39:2099)

6. B. Solomon, Tantrices of spherical curves. Amer. Math. Monthly, 103(1):30-39, 1996. MR1369149(97a:53002)

7. S. Tabachnikov, On skew loops, skew branes, and quadratic hypersurfaces. Moscow Math. J., 3 (2003), 681-690. MR2025279 (2004m:53007) 
8. J. H. White, Global properties of immersions into Euclidean spheres. Indiana Univ. Math. J., 20:1187-1194, 1971. MR0283815 (44:1045)

9. Y.-Q. Wu, Knots and links without parallel tangents. Bull. London Math. Soc., 34:681-690, 2002. MR.1924195 (2003h:57012)

School of Mathematics, Georgia Institute of Technology, Atlanta, Georia 30332

Current address: Department of Mathematics, Penn State University, University Park, Pennsylvania 16802

E-mail address: ghomi@math.gatech.edu

$U R L$ : www. math.gatech.edu/ ghomi 